\title{
An efficient modeling and simulation of quantum key distribution protocols using OptiSystem $^{\text {TM }}$
}

\begin{abstract}
In this paper, we propose a modeling and simulation framework for quantum key distribution protocols using commercial photonic simulator OptiSystem $\hat{E}$. This simulation framework emphasize on experimental components of quantum key distribution. We simulate BB84 operation with several security attacks scenario and noise immune key distribution in this work. We also investigate the efficiency of simulator's inbuilt photonic components in terms of experimental configuration. This simulation provides a study to analyze the impact of experimental photonic components in quantum key distribution process.
\end{abstract}

Keyword: Quantum cryptography; QKD-simulation; OptiSystem 